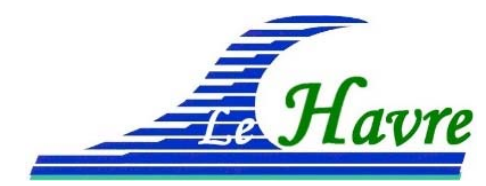

XVI ${ }^{\text {èmes }}$ Journées Nationales Génie Côtier - Génie Civil

Le Havre, 2020

DOI:10.5150/jngcgc.2020.009 (C) Editions Paralia CFL

disponible en ligne - http://www.paralia.fr - available online

\title{
Optimisation du système public d'information sur les données de houle côtières, CANDHIS
}

\author{
Xavier KERGADALLAN ${ }^{1}$, Sébastien DUPRAY ${ }^{1}$, Nathalie METZLER ${ }^{1}$
}

1. Cerema, direction technique Eau, mer et fleuves, 134 rue de Beauvais, CS 60039, 60280 Margny-lès-Compiègne, France.

CANDHIS.DTecEMF@cerema.fr

\section{Résumé :}

Le Cerema, au travers des précédentes entités qui le composent, gère depuis 1972 un ensemble de stations côtières de mesure de houle le long du littoral français. Le réseau est à la fois un réseau de mesure opérationnel ainsi qu'un système d'information national qui forment un tout cohérent, nommé CANDHIS (Centre d'Archivage de Données de Houle In Situ). Il s'est progressivement étendu par opportunités successives pour atteindre, à ce jour, 34 stations de mesure en métropole et outre-mer. Ainsi, CANDHIS met à disposition des données indispensables à divers domaines d'activités (sécurité maritime, développement des énergies marines, gestion des infrastructures maritimes et portuaires, gestion des risques et des territoires littoraux, ...) à des fins opérationnelles d'intervention publique comme privée, de recherche ou régaliennes.

Afin d'étudier les limites locales d'utilisation des données CANDHIS et d'orienter sa politique d'instrumentation, le Cerema a effectué une analyse spatiale des données de vagues. La méthode mise en œuvre s'appuie sur une analyse des simulations numériques ANEMOC 2, avec le calcul de facteur de corrélation entre la localisation des houlographes et l'ensemble des points de simulation ANEMOC.

Le résultat de cette analyse permet d'objectiver la qualité de la couverture du littoral par le réseau. Ces éléments scientifiques et techniques alimentent actuellement les réflexions au niveau national sur son optimisation.

\section{Mots-clés :}

CANDHIS, Houlographe, Vagues, Houle, États de mer, Mesure in situ, Bouée de mesure, Réseau, ANEMOC, Métropole.

\section{Des services opérationnels pour les acteurs maritimes et côtiers}

\subsection{Des données aux services d'usages toujours plus variés}

La connaissance du régime des états de mer au large et à la côte est importante voire indispensable dans de nombreux domaines (CEREMA, 2018) et notamment les risques littoraux, l'étude de l'évolution du climat, le suivi du trait de côte, le dimensionnement d'ouvrages portuaires (HAMM, 2008), la sécurité de la navigation, ... 


\section{Thème 1 - Hydrodynamique côtière}

Pour caractériser les climats de vagues (saisonnalités, directions, hauteurs et périodes caractéristiques des vagues) (AIPCN-AIRH, 1986; CEREMA, 2020), on utilise classiquement les mesures in situ (bouée de mesure, capteur acoustique, perche à houle...) et des observations satellitaires (capteurs altimétriques, radars) (TUCKER \& PITT, 2001). Dans la zone littorale, caractérisée par des variations rapides et/ou importantes de la bathymétrie, l'évolution de la houle (GODA, 2000) est complexe et la mesure in situ est le moyen le plus approprié pour obtenir une information ponctuelle de qualité (ARDHUIN et al., 2019). Par ailleurs, la mesure in situ est nécessaire pour compléter et caler les informations spatiales obtenues par la modélisation numérique comme par la télémesure par satellite.

Le Cerema, au travers des précédentes entités qui le composent, gère depuis 1972 un ensemble de stations de mesure de houle le long du littoral français et le système d'information sur les données associées (concentration, contrôle et traitement, et diffusion des données) (SERVICE TECHNIQUE DES PHARES ET BALISES, 1978). L'ensemble forme le Centre d'Archivage National des Données de Houle In Situ, CANDHIS.

Le réseau s'est développé au fil du temps pour atteindre 34 stations de mesure en métropole et outre-mer. Les stations CANDHIS sont toutes équipées d'une bouée dédiée à la mesure de houle. La localisation des points de mesure CANDHIS est un subtil compromis entre le besoin de l'investisseur premier, le besoin des utilisateurs de la donnée et les contraintes de site. Le réseau CANDHIS étant présent sur globalement l'ensemble des façades maritimes, la qualité de la couverture du réseau est un enjeu important à objectiver pour le choix des futures zones à instrumenter et la pertinence d'un redéploiement de certaines stations. Les connexions à CANDHIS sont d'environ 200000 par an pour les données en temps réel et d'environ 140 demandes de données analysées en temps différé, avec des usages toujours plus variés.

\subsection{Une couverture spatiale historique et différents modes de gestion}

Le nombre de stations de mesure actuellement en métropole et outre-mer est de 34 stations (dont celle de Gravelines déployée courant 2020).

La localisation des points de mesure est décidé au niveau local en fonction du besoin de l'organisme assurant l'investissement (une collectivité, un port, une entreprise...). Elle est aussi discutée au national en fonction des enjeux avec le Cerema, Météo-France (plus particulièrement depuis le développement de la vigilance vagues submersions), le Shom et les administrations centrales. Même si le Cerema apporte un appui dans le choix des équipements pouvant ensuite être producteur de données pour le réseau CANDHIS, il n'existe aucun référentiel technique obligatoire.

La localisation des stations de mesure CANDHIS doit aussi répondre à différentes contraintes en termes de : 


\section{XVIèmes Journées Nationales Génie Côtier - Génie Civil \\ Le Havre 2020}

a) Distance à la côte : la bouée de mesure doit être suffisamment près de la côte pour être représentative des vagues se propageant à la côte, mais suffisamment loin pour être représentative d'un large linéaire côtier.

b) Paramètres océanographiques : il faut prendre en compte les courants et la profondeur pour la tenue et le dimensionnement du matériel.

c) Activité maritime : elle est à l'origine du principal risque de perte du matériel. Il convient donc de s'écarter autant que possible des routes régulières, mais aussi des zones de pêches ou des zones de navigation de plaisance.

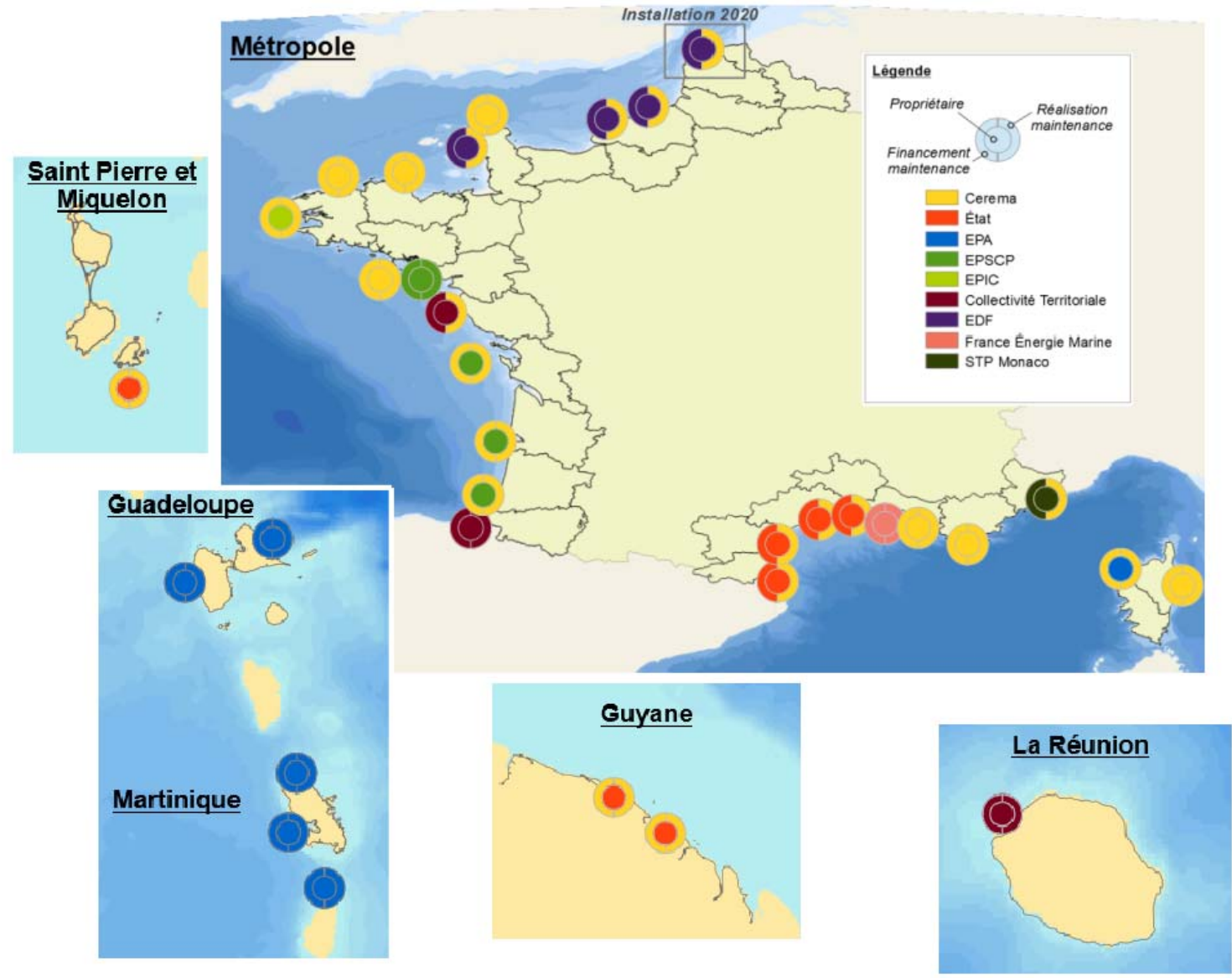

Figure 1. Localisation et mode des gestions des stations de mesure de houle CANDHIS.

Cette équation est souvent complexe à résoudre. L'implantation d'un point de mesure fait l'objet d'un compromis entre ces différentes contraintes.

La carte d'implantation des différentes stations de mesure et leur mode de gestion est présentée sur la figure 1. 


\section{Thème 1 - Hydrodynamique côtière}

\section{Méthode d'analyse de la couverture spatiale}

\subsection{Principe de base de la méthode d'analyse}

Dans le cadre du présent article, l'analyse de la couverture spatiale du réseau CANDHIS a consisté à étudier si en un point donné (nommé ci-après "point d'analyse »), les observations au point de mesure CANDHIS le plus proche (nommé ci-après « point de référence ») sont suffisantes pour décrire de manière satisfaisante les états de mer en ce point donné.

Il est fait le choix d'utiliser la base de données d'états de mer ANEMOC-2 (Cerema et EDF R\&D-LNHE), afin d'avoir des données aux points d'analyse et aux points de référence. ANEMOC-2 est une base de simulations numériques sur la période de 1979 à 2010, sur les façades métropolitaines (LAUGEL et al., 2014), mais aussi la Réunion et les Antilles.

Il est aussi fait le choix de ne s'intéresser qu'à la hauteur significative des vagues (Hs) pour décrire de manière synthétique les états de mer, lors des seuls événements tempétueux. De plus l'analyse des paramètres des états de mer se focalise aux événements tempétueux. Nous considérons ici que Hs est un paramètre caractéristique d'état de mer très largement utilisé (DEAN \& WALTON, 2009; EUROTOP, 2016) et que les événements de tempête sont critiques dans les études hydrauliques maritimes (CETMEF, 2013 ; MEDDE DGPR SRNH 2014]. Ce choix est toutefois à l'origine d'une limite de la méthode (discutée au §3.2).

Les différentes étapes de la méthode de calcul sont les suivantes :

a) Analyse en utilisant les houlographes en points de référence (assimilés au point ANEMOC le plus proche) et l'ensemble des points ANEMOC en points d'analyse.

b) Sélection automatique des pics de tempêtes au point de référence (Hs supérieure au percentile 99,5\%, indépendance des pics de tempêtes à $+/-12$ heures).

c) Sélection au point d'analyse de la Hs correspondant aux pics de tempête (Hs maximum sur $+/-12$ heures autour du pic de tempête).

d) Calcul de l'incertitude du facteur de corrélation entre le point de référence et le point d'analyse. Le travail est effectué en valeurs relatives (pourcentage de dispersion des Hs autour de la valeur moyenne).

e) Les valeurs d'incertitude calculées sont utilisées pour définir les zones d'influence. Plus l'incertitude « relative » est faible, plus la corrélation entre les deux points est jugée bonne.

\subsection{Exposition aux tempêtes}

Par contre, toutes les tempêtes touchant le houlographe (point de référence) peuvent atteindre le site distant (point d'analyse) sans que l'inverse soit vrai. L'incertitude calculée sera faible alors que logiquement les Hs ne devraient pas être bien corrélées. 


\section{XVIèmes Journées Nationales Génie Côtier - Génie Civil \\ Le Havre 2020}

Pour pallier ce problème, une analyse inverse est aussi effectuée en utilisant les points ANEMOC en référence et les houlographes en tant que points d'analyse. Les incertitudes calculées sont toujours positionnées aux points ANEMOC.

L'interprétation se fait en croisant le résultat de l'analyse avec le houlographes en points de référence (une première valeur d'incertitude), avec le résultat de l'analyse inverse avec les points ANEMOC en points de référence (une deuxième valeur d'incertitude).

\subsection{Seuil d'incertitude}

Chaque point ANEMOC est donc caractérisé par deux valeurs incertitudes. La fixation d'un seuil d'incertitude permet de définir des zones d'influence des houlographes. Un point ANEMOC est considéré comme étant dans la zone d'influence du houlographe si ses deux valeurs d'incertitude sont en dessous du seuil fixé. Si un point ANEMOC est sous l'influence de deux houlographes, il y a recouvrement de zone d'influence.

Le seuil d'incertitude est fixé à dire d'expert, simultanément sur l'ensemble de la zone d'étude. Il résulte d'un juste équilibre entre la recherche de jonction des zones d'influence entre houlographes voisins (augmentation du seuil) et la minimisation des zones de recouvrement (diminution du seuil). Il est fixé ici à $\pm 20 \%$, soit une dispersion de $\pm 20 \%$ des Hs aux points ANEMOC pour une Hs donnée au houlographe, et réciproquement.

\section{Analyse et perspectives}

\subsection{Principaux résultats}

Le résultat se présente sous la forme d'une carte de zones d'influence des houlographes. Cette carte est présentée figure 2. Les zones d'influence ont pu être établies pour la métropole, la Guadeloupe, la Martinique et la Réunion.

Les points ANEMOC sont matérialisés par de petits disques de couleur blanche, verte ou rouge :

a) Blanche si au moins une des deux incertitudes calculées (voir §3.1) est au-dessus du seuil fixé. Les Hs sont jugées peu corrélées aux Hs du houlographe le plus proche.

b) Verte signifie si les deux incertitudes calculées sont en dessous du seuil fixé. Les Hs sont jugées corrélées aux Hs du houlographe le plus proche.

c) Rouge si les Hs sont jugées corrélées aux Hs d'au moins deux houlographes. Il y a donc recouvrement de zones d'influence.

Les houlographes sont représentés par des disques de couleur bleue ou jaune :

a) Bleue si le houlographe est géré dans le cadre d'un partenariat local.

b) Jaune si le Cerema gère le houlographe et peut décider d'ajuster sa position. Dans le cadre d'une réflexion globale d'optimisation spatiale, seul le redéploiement des houlographes en jaune peut être étudié.

La constitution au fil du temps a malgré tout permis de constituer un réseau offrant une couverture globalement cohérente. 


\section{Thème 1 - Hydrodynamique côtière}

Sur le littoral Manche Atlantique, la couverture présente des lacunes en baie de Seine, baie de Saint-Brieuc et baie d'Audierne. Pour Saint-Brieuc et Audierne, il peut être envisagé de redéployer les houlographes de Belle-Ile et de Bréhat. Pour la baie de Seine, il conviendrait d'installer une nouvelle station de mesure.

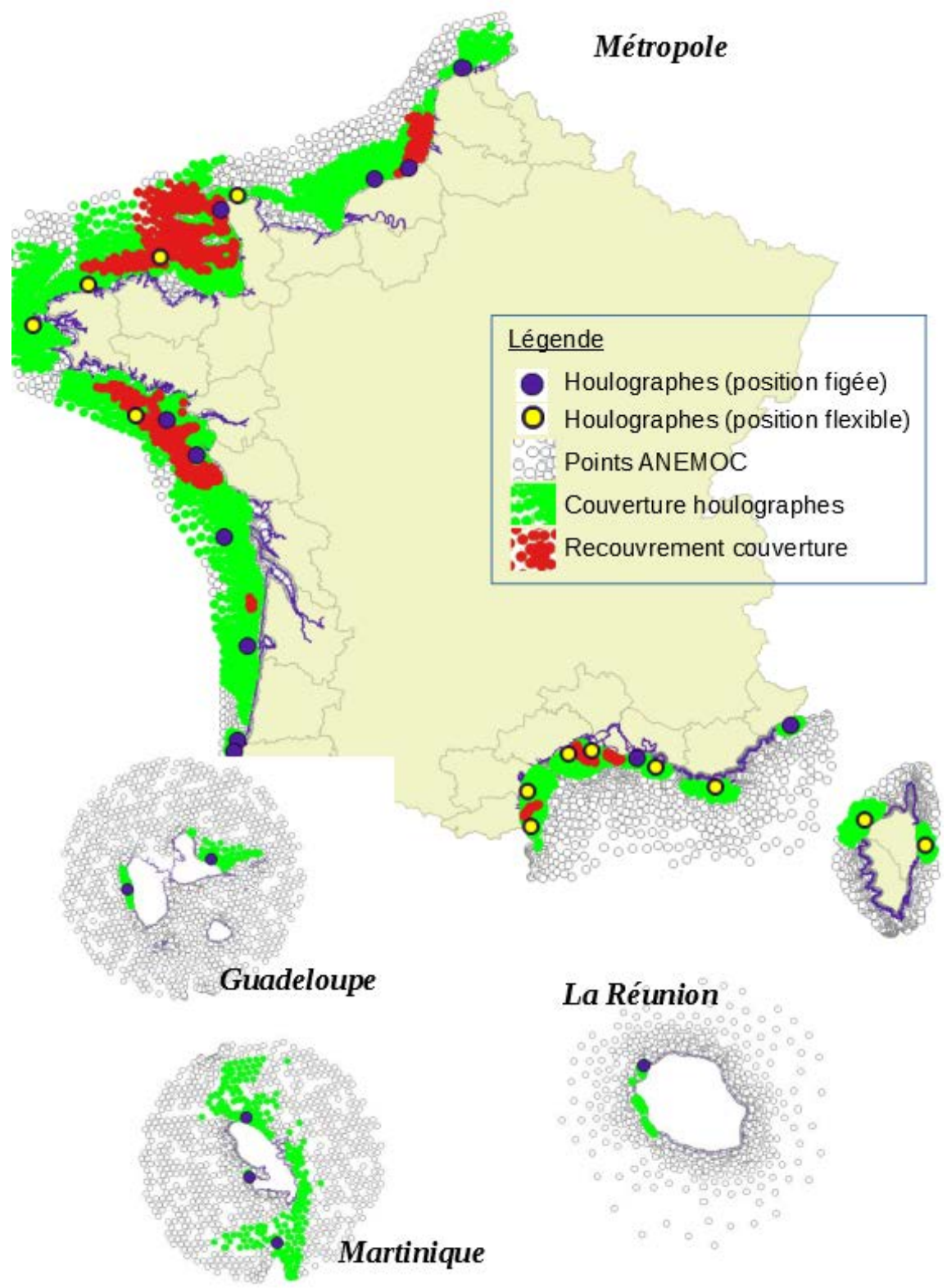

Figure 2. Cartographie de la couverture du réseau de mesure de houle CANDHIS.

Sur le littoral Méditerranéen, la couverture présente des lacunes sur le littoral est (entre l'île de Porquerolles et Monaco) et sur la Corse (au nord, sud-est et sud-ouest). 


\section{XVI'̀mes Journées Nationales Génie Côtier - Génie Civil \\ Le Havre 2020}

Il serait nécessaire d'installer 4 nouvelles stations de mesure, dont 3 pour la seule Corse. Un redéploiement serait possible en couvrant le golfe du Lion (ouest méditerranée) avec 3 houlographes au lieu des 4 actuels.

En Guadeloupe seules les façades est et ouest sont couvertes. Du fait de la bathymétrie du site, les façades nord et sud ne sont pas couvertes, mais cette portion du littoral est moins exposée aux fortes vagues.

En Martinique seule la façade ouest, la plus exposée aux fortes vagues, est couverte. Il existe bien un houlographe à l'ouest de Fort-de-France, mais l'influence de la baie sur les cyclones en provenance de l'ouest rendrait les observations au houlographe assez différentes de celle du reste du littoral ouest.

À la réunion seule la façade ouest est couverte. Il serait nécessaire de disposer de trois autres points de mesure afin de couvrir l'ensemble du littoral.

\subsection{Analyse critique de la méthode employée et pistes d'amélioration}

L'analyse a été réalisée sur le seul paramètre de Hs spectrale (Hm0). Mais d'autres paramètres sont très utilisés dans les études de vagues : des paramètres sur les hauteurs $\left(\mathrm{H}_{1 / 3}, \mathrm{Hrms}, \mathrm{Hmax} ..\right)$, sur les périodes $\left(\mathrm{T}_{\mathrm{H} 1 / 3}, \mathrm{~T}_{01}, \mathrm{~T}_{02}, \mathrm{TE} \ldots\right)$, sur les directions (Dir pic, Dir moy, Etal pic, Etal, moy...) etc. L'analyse s'est aussi focalisée sur les événements tempête (définis arbitrairement à partir d'un seuil sur les quantiles). Mais certaines études sont basées la climatologie annuelle ou sur les événements extrêmes (décennaux, centennaux...). Pour être complet, il conviendrait donc de refaire l'analyse sur l'ensemble des paramètres, sur l'ensemble des hauteurs, et de croiser les résultats avec une méthode qui reste à développer.

Les limites des zones d'influence et de recouvrement sont très dépendantes du seuil fixé sur les incertitudes.

L'analyse s'appuie sur les données issues d'un modèle numérique d'états de mer (ANEMOC-2), qui lui-même a besoin de mesure in situ pour se caler.

Un objectif essentiel de CANDHIS est de constituer de longues séries de mesure, le redéploiement d'une station de mesure est donc plutôt à éviter.

$\mathrm{Au}$ vu de l'ensemble des limites présentées ci-dessus, la présente analyse doit être considérée comme une approche qualitative de la qualité de la couverture du réseau CANDHIS. C'est néanmoins un outil d'aide à la décision utile par exemple pour la gestion du réseau. Son interprétation ne peut se faire qu'en croisant avec une cartographie des enjeux sur le littoral.

\section{Conclusion et perspectives}

CANDHIS a pour objectif depuis 1972 la mesure et la diffusion de données de houle in situ dans le domaine côtier. Avec 34 stations de mesure en 2020 sur l'ensemble du littoral (métropole et outre-mer), et la constitution historique en opportunité du réseau, se pose la question de l'optimisation de sa couverture. 


\section{Thème 1 - Hydrodynamique côtière}

Le Cerema a ainsi conduit une analyse originale basée sur des données issues de simulations numériques, les données ANEMOC. Un calcul d'incertitude de corrélation entre les hauteurs significatives des vagues au houlographe et sur l'ensemble des points voisins, a permis de dresser une cartographie des zones d'influence des houlographes sur l'ensemble du littoral. Au vu des limites de la méthode, cette analyse doit être considérée comme un outil d'aide à la décision et non comme un résultat absolu en soi.

Cette analyse montre globalement une couverture de bonne qualité à l'échelle du réseau et permet d'identifier des faiblesses localisées en Normandie, Bretagne, côte d'Azur, Corse et Réunion. À cela s'ajoute les secteurs pour lesquels CANDHIS n'est pas présent (le nord des Antilles, Mayotte, Wallis et Futuna, la Nouvelle-Calédonie et la Polynésie Française).

Ce travail effectué en 2019 a permis d'orienter les choix stratégiques de développement du réseau CANDHIS en métropole. Pour 2020, grâce au soutien particulier du ministère de la Transition écologique et solidaire (Direction Générale de la Prévention des Risques), le Cerema a prévu d'installer trois nouvelles stations de mesure : une en baie de Seine, une autre entre Porquerolles et Monaco et la dernière au sud-ouest de la Corse. Ces choix ont été effectués en croisant le résultat de la présente analyse avec une estimation des enjeux à la côte.

À plus long terme, une réflexion s'engage pour moderniser le système d'information qui est le support à la diffusion des données, ainsi que sa gouvernance dans une recherche de synergie renforcée avec les bénéficiaires des données afin de garantir dans la durée un service de qualité.

\section{Références bibliographiques}

AIPCN-AIRH (1986). Paramètres des états de mer. Bulletin de l'association internationale permanente des congrès de navigation, 49 p., supplément au bulletin $\mathrm{n}^{\circ} 52$, 1986.

ARDHUIN F., STOPA J.E., CHAPRON B., COLLARD F., HUSSON R., JENSEN R.E., JOHANNESSEN J., MOUCHEL A., PASSARO M., QUARTLY G.D., SWAIL V., YOUNG I. (2019). Observing sea states. Front. Mar. Sci. 6:124, https://doi.org/10.3389/fmars.2019.00124

CEREMA (2018). Etudes hydrauliques maritimes - Guide à destination des maîtres d'ouvrage, Édition Cerema, Collection références, $156 \mathrm{p}$.

CEREMA (2020). Fiches synthétiques de mesure des états de mer du réseau CANDHISMAJ 2020. Édition Cerema, Collection Données, 3 tomes.

CETMEF (2013). Analyse statistique des niveaux d'eau extrêmes, environnements maritime et estuarien. Édition Cerema, Collection référence.

DEAN R.G., WALTON T.L. (2009). Wave setup. In handbook of coastal and ocean engineering. Young C. Kim ed., World scientific, pp 1-23. 


\section{XVIèmes Journées Nationales Génie Côtier - Génie Civil \\ Le Havre 2020}

EUROTOP, VAN DER MEER J.-W., ALLSOP N.W.H., BRUCE T., DE ROUCK J., KORTENHAUS A., PULLEN T., SCHUTTRUMPF H., TROCH P., ZANUTTGH B. (2016). Manual on wave overtopping of sea defences and related structures, www.overtopping-manual.com.

GODA Y. (2000). Random seas and design of maritime structures. World scientific publishing, Singapore, $3^{\text {rd }}$ edition, Advanced Series on Ocean Engineering, Vol. 33, 732 p. https://doi.org/10.1142/7425

HAMM L. (2008). Calculs d'agitation portuaire, état de l'art. $7^{\text {èmes }}$ Journées Scientifiques et Techniques du Cetmef, Hydraulique portuaire, 8 décembre 2008, Paris. LAUGEL A., TIBERI-WADIER A.-L., BENOIT M., MATTAROLO G. (2014). Anemoc-2 Atlantique et Méditerranée : calibration et validation de deux nouvelles bases d'état de mer construites par simulations numériques rétrospectives sur 1979-2010,

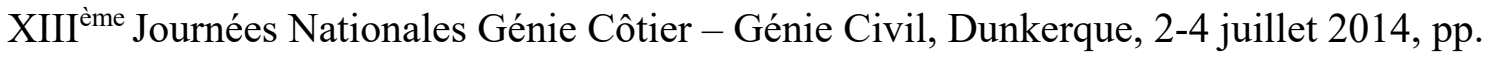
109-120. https://doi.org/10.5150/jngcgc.2014.013

MEDDE DGPR SRNH (2014). Guide méthodologique : Plan de prévention des risques littoraux.

SERVICE TECHNIQUE DES PHARES ET BALISES (1978). Mesure de la houle. Analyse vague par vague. Résultats de traitement des observations. Édition STPB.

TUCKER M.J., PITT E.G. (2001). Waves in ocean engineering. Elsevier Ocean Engineering Book Series, Vol. 5, 548 p. 
Thème 1 - Hydrodynamique côtière 